\title{
Charm production by neutrinos in the CHORUS experiment
}

\section{A. Murat Güler* for the CHORUS collaboration}

\author{
Nagoya University, Furo-cho, chikusa-ku, Nagoya, 464-8602, Japan
}

E-mail: 'Alìi.Murat. Guler@cern.chi

\begin{abstract}
We report a measurement of the $D^{0}$ production rate in $\nu_{\mu}$ charged current interactions. Recent improvements of automatic scanning systems and methods allow the CHORUS experiment at CERN to study charm production with a sub-micron spatial resolution typical for emulsion, leading to very small backgrounds. Based upon $282 D^{0}$ decays with an estimated background of $9.2 \pm 1.4 K_{s}^{0}$ and $\Lambda^{0}$ found in 25693 located $\nu_{\mu}$ interactions we obtain a $D^{0}$ production rate in $\nu_{\mu}$ charged current interactions $1.98 \pm 0.13$ (stat.) \pm 0.11 (syst.) $\%$ at $27 \mathrm{GeV}$ average $\nu_{\mu}$ energy.
\end{abstract}

\section{Introduction}

In neutrino deep inelastic scattering (DIS), a charmed hadron is produced when a neutrino interacts with a strange (s) sea quark or valance down (d) quark, producing a charm (c) quark. The subsequent fragmentation leads to a charmed hadron, mostly D mesons through the hadronization process. The Feynman diagram for this process is shown in figure 1.

After the first observation [i] $\left.\bar{i}_{1}\right]$ of single charm particle production in neutrino interactions, several experiments [2] have studied charmed particle production properties mostly by analyzing opposite sign dimuon events. In these events, the decay of a charmed particle is not seen directly, its presence is inferred from the observation of opposite sign dimuon in the final state. Experiments of this type, however, suffer from a large background of events in which the second muon originates from an undetected decay in flight of a pion or a kaon rather than from a charmed decay. Nevertheless these experiments



Figure 1: Neutrino charm production in DIS. have provided measurements of the strange quark content of the nucleon as well as an estimation of the charm quark mass. On the other hand, in order to improve the knowledge of

\footnotetext{
${ }^{*}$ Speaker.
} 
neutrino charm production, one needs an unbiased sample of charm events. Experiments using nuclear emulsion have a sub-micron spatial resolution which allows $3 \mathrm{D}$ reconstruction of events. Therefore, it can be used for topological identification of short lived particles

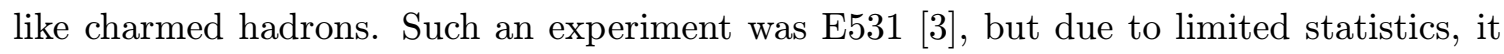
could provide only qualitative information. The recent development of automatic scanning devices within CHORUS has made studies of charm production with high statistics possible.

\section{The CHORUS experiment}

The CHORUS detector is a hybrid setup that combines the nuclear emulsion with various electronic detectors: a scintillating fiber tracker, trigger hodoscopes, a hadron spectrometer, a calorimeter and a muon spectrometer.

The nuclear emulsion acts as target and simultaneously, as a detector for neutrino interactions. The emulsion target which is segmented into 4 stacks has an overall mass of $770 \mathrm{~kg}$. Each stack is followed by three interface emulsion sheets and by a set of scintillating fiber tracker planes. The interface sheets and the fiber trackers provide accurate particle trajectory predictions into the emulsion stack in order to locate the vertex positions. The emulsion scanning has been performed by fully automatic microscopes equipped with

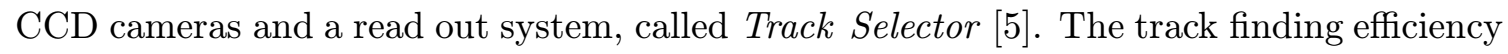
of the track selector is higher than $98 \%$ for track slopes less than $400 \mathrm{mrad}$.

The West Area neutrino Facility (WANF) at CERN provides a neutrino beam of 27 $\mathrm{GeV}$ average energy consisting mainly of $\nu_{\mu}$ with a $5 \% \bar{\nu}$ contamination. During four years (1994-1997) of operation CHORUS recorded about $10^{6}$ neutrino interactions originating in the emulsion target. Of these, 170,000 have been located and analyzed for $\nu_{\mu} \rightarrow \nu_{\tau}$

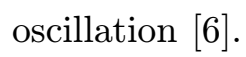

\section{Analysis}

The CHORUS experiment is mainly dedicated to the $\nu_{\mu} \rightarrow \nu_{\tau}$ oscillation search, hence, the data selection $[\bar{i} \bar{i}]$ for the first phase of the analysis was optimized towards the detection of $\tau$ decaying into a single charged particle. In particular, only events with a muon momentum less than $30 \mathrm{GeV} / c$ were selected for emulsion scanning. This cut reduces by $29 \%$ the number of events to be scanned. On the other hand, it rejects $15 \%$ of the $\nu_{\tau}$ interactions if the $\nu_{\tau}$ has the same energy spectrum as $\nu_{\mu}$. Therefore, it has a marginal effect on the $\tau$ sensitivity but plays a more important role in the present analysis of charm production. In order to correct for this cut about $3 \mathrm{~K} \nu_{\mu} \mathrm{CC}$ events with a muon momentum greater than $30 \mathrm{GeV} / c$ were located in addition and analyzed.

In order to detect the decay topologies in the emulsion target, a new method called "netscan" [i]" has been applied. A fiducial volume of $1.5 \times 1.5 \times 6.3 \mathrm{~mm}^{3}$ centered on the located vertex is scanned and all the track segments within angular acceptance $\left(\left|\theta_{y, z}\right|<400\right.$ $\mathrm{mrad}$ ) are recorded. The scanning of an event takes $11 \mathrm{~min}$ with the latest version of the Track Selector, UTS. Typically, about $5 \mathrm{~K}$ track segments are recorded per event and their 
parameters (3 position coordinates and 2 angles etc.) are stored in a data base. From this huge number of track segments, tracks are reconstructed offline. Tracking and vertex reconstruction involve a number of steps: first, the global (plate by plate) and the local alignment (event by event) have been done by comparing the pattern of segments in a given plate with corresponding pattern in the next plate. Then, tracking is done by extrapolating the track segments on the downstream and upstream plate and searching for candidates for connections. After connection of all matched segments, a second, more accurate, alignment of the plates has been done using tracks passing through the entire volume. These are mainly due to muons associated with the neutrino beam or nearby test beams. After the fine alignment, the residual of the segment positions with respect to the fitted track is about $0.45 \mu \mathrm{m}$. After the final alignment, about 400 tracks remain in the fiducial volume. The vast majority can be recognized as due to low energy (typically less than $100 \mathrm{MeV}$ ) particles and discarded. Moreover, a $\chi^{2}$ cut is applied to select the "good" tracks. The final step is the rejection of tracks passing through the scanning volume. After this filtering, the mean number of tracks originating in the scan volume is about 40. Finally, tracks are associated into vertices using the distance of closest approach.

\subsection{Event selection}

Tracks originating from the interaction and stopping in the scanning volume are candidates for decays. In order to select interesting decay topologies, various cuts are applied. They are optimized in order to keep the eyescan load reasonable, while keeping high efficiency for decay finding. The following requirements were used

\begin{tabular}{cc|cc}
\hline Accepted events & & Rejected events & \\
\hline V2 & 225 & low momentum & 174 \\
V4 & 57 & hadron int. & 68 \\
C1 & 121 & e-pair & 42 \\
C3 & 124 & -ray & 2 \\
C5 & 7 & other & 30 \\
V2+C1 & 1 & & \\
\hline Total & 535 & & 316 \\
\hline
\end{tabular}

Table 1: Eye-scan results of candidates.

- the primary muon and at least one of the stopping tracks in the scanning volume were reconstructed with more than one track segment and matching at the $99 \%$ confidence level the direction of a track reconstructed in the fiber tracker system.

- the impact parameter of at least one of the stopping tracks to the vertex point must be larger than $\sqrt{3^{2}+(2 \sigma d x)^{2}} \mu \mathrm{m}$ where $\sigma=\sqrt{0.00305^{2}+(0.0194 \theta)^{2}}$ is the parametrization of the angular error and $d x$ is the distance (in the beam direction) of the vertex to the most upstream daughter track segment. This impact parameter must be also smaller than $400 \mu \mathrm{m}$. This last cut mainly rejects tracks not related to the neutrino interaction.

The analysis presented here is based on 25,693 netscanned events. The selection criteria retained 851 candidates for the charm decays. In order to confirm the decay topology, these candidates were checked by eye. The result of the eye-scan is given in the Table 1 . The purity of the automatic selection is $63 \%$. The observable decay topologies are classified as odd-prong decays of a charged particle (mainly $D^{+}, D_{s}^{+}, \Lambda_{c}^{+}$) or even-prong decays of a 
neutral particle (mainly $D^{0}$ ). These are denoted in Table 2 as V2, V4 or V6 for neutral and C1, C3 or C5 for charged decays according to the prong multiplicity. There is also one event having both V2 and $\mathrm{C} 1$ decay topologies denoted as $\mathrm{V} 2+\mathrm{C} 1$. This event is a candidate of associated charm production; further analysis is underway. The rejected sample consists mainly of hadronic interactions, delta rays or gamma conversions $(\sim 36 \%)$ and of low momentum tracks which, due to multiple scattering, appear as tracks with a large impact parameter $(\sim 54 \%)$. The remaining $10 \%$ consists of fake vertices, reconstructed using one or more background tracks.

The confirmed $D^{0}$ sample contains 282 candidates. For 33 of these events (24 V2 and $9 \mathrm{~V} 4$ ), the muon momentum is greater then $30 \mathrm{GeV} / c$.

\subsection{Monte-Carlo simulation}

$D^{0}$ detection efficiency can be split into two terms: the first term is the location efficiency of $\nu_{\mu} \mathrm{CC}$ events, given by the efficiency of the reconstruction of the neutrino interaction and of its location in emulsion. The second term is the efficiency of the decay search, the netscan method.

In order to estimate efficiencies and backgrounds, a Monte-Carlo simulation with the complete set-up and event reconstruction has been performed. The location efficiencies are found to be $59.0 \pm 0.2 \%$ and $51.4 \pm 0.4 \%$ for all $\nu_{\mu} \mathrm{CC}$ events and $\mathrm{D}^{0}$ events respectively. In order to estimate the efficiency of netscan procedure, one needs to reproduce realistic conditions of track density. To achieve this, MC tracks are simulated in the emulsion and then merged with real netscan data. The combined data is then passed through the same reconstruction and selection programs as used for real data.

The systematic uncertainties of netscan efficiency arise mainly from the choice of the event generator and from the variation in the emulsion data quality. The first source of systematic error is estimated to be $3.4 \%$ by comparing results obtained with different structure functions and fragmentation functions. The second one is estimated to be $2 \%$ by merging simulated netscan data with different sets of spurious netscan data corresponding to different track densities and different alignment accuracies. The mean netscan efficiency for the $D^{0}$ decay search is $58.6 \pm 4.0 \%$ for V2 and $70.1 \pm 4.2 \%$ for V4, where errors combine statistical and systematical uncertainties.

By processing $\nu_{\mu} \mathrm{CC}$ interactions with no $D^{0}$ in the final state through the same chain of programs, the background rate is evaluated to be $(3.6 \pm 1.0) 10^{-4}$ per located CC event. In the present sample of 25,693 events this corresponds to $9.2 \pm 2.6$ background events, mainly $K_{s}^{0}$ and $\Lambda^{0}$ decays.

\section{4. $D^{0}$ production rate}

Taking into account the estimated efficiencies and background, one obtains a $D^{0}$ production rate relative to $\nu_{\mu} \mathrm{CC}$ interactions: $\frac{\sigma\left(D^{0}\right)}{\sigma(C C)}=1.98 \pm 0.13$ (stat. $) \pm 0.11($ syst. $) \%$ in good agreement with the result of E531 [i3] (dashed lines in Figure 2) based on a much lower statistics. The topological ratio V4/V2 is found to be $23.3 \pm 4.4 \%$ in agreement with the world average value $20.1_{-1.9}^{+2.7} \%\left[\overline{9}\left[\right.\right.$. The dependence of the $D^{0}$ production rate in the 
measured neutrino energy is shown in Figure 2. A second phase of analysis of the CHORUS data has been started, with upgraded reconstruction codes and scanning systems. In about one year, it is expected collect an unbiased sample of 3000 charm events will be collected, allowing a detailed and almost background-free study of all the charm production processes.

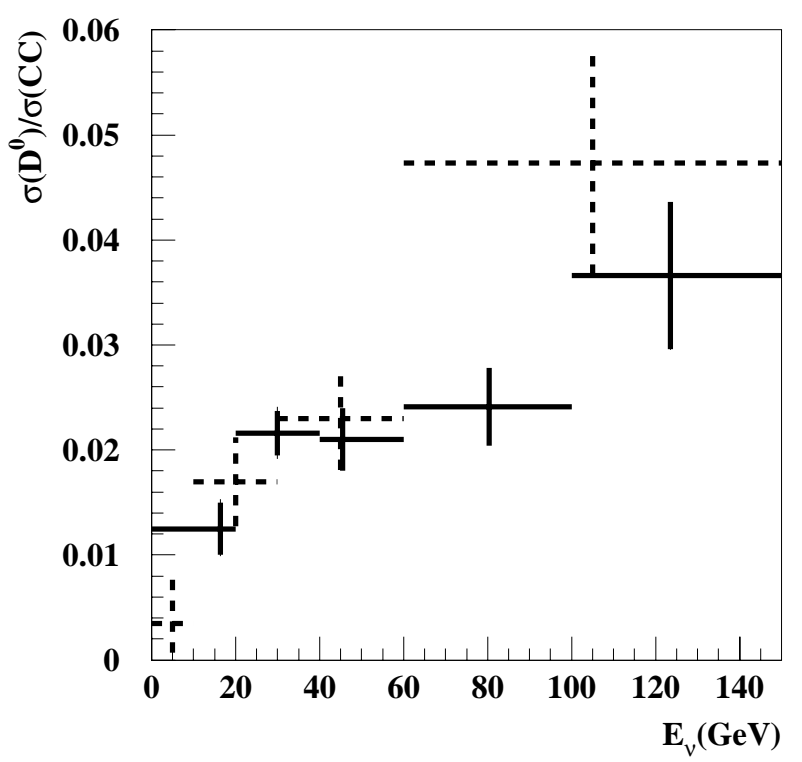

Figure 2: $D^{0}$ production rate as a function of neutrino energy. CHORUS result is shown as solid lines. Also E531 result is shown (dashed lines).

\section{References}

[1] A Benvenuti et al., Phys. Rev. Lett. 34, 419 (1975).

[2] H. Abramowicz et al., Z. Phys., C15 19 (1982), S.A. Rabinowitz et al., Phys. Rev. Lett. 70, 134 (1993), M. Jonker et al., Phys. Lett. B107 241 (1981), P. Vilain et al., Eur. Phys. J. C11 19 (1999), P. Astier et al., Phys. Lett. B486 35 (2000).

[3] N. Ushida et al., E531 Collaboration, Phys. Lett. B206, 375 (1988).

[4] E. Eskut et al., CHORUS Collaboration, Nucl. Instr. and Meth. A401 7 (1997).

[5] T. Nakano, PhD thesis, Nagoya University, Japan (1997).

[6] E. Eskut et al., CHORUS Collaboration, Phys. Lett. B503 1 (2001).

[7] K. Kodama et al., DONUT Collaboration, Phys. Lett. B504 218 (2001).

[8] M. Güler, PhD thesis, M.E.T.U., Turkey (2000).

[9] Particle Data Group, Euro. J 15, (2000). 